\title{
Lipoid proteinosis presenting with neuropsychiatric manifestations
}

\author{
ROBIN A EMSLEY, LOUIS PASTER \\ From the Department of Psychiatry, Faculty of Medicine, University of Stellenbosch, South Africa
}

SUMMARY Two patients with lipoid proteinosis are reported in whom paranoid symptoms were the presenting feature. Both had a long standing impairment of memory and bilateral medial temporal lobe calcification demonstrated by CT scan. Possible associations between the anatomical site of these lesions and the neuropsychiatric manifestations are discussed.

Lipoid proteinosis (Urbach-Wiethe disease) is a rare, hereditary, systemic disorder characterised by widespread deposition of storage material, particularly in the skin and mucous membranes of the mouth, pharynx and larynx. Bilateral oval calcification in the region of the medial temporal lobes has been found in $52 \%$ of cases ${ }^{1}$ and seems to be unique to this condition. The disease is inherited as an autosomal recessive trait, ${ }^{1}$ and most patients appear to be of European descent. It has been reported most often in South Africa, where the responsible gene was introduced in the mid-17th century by a German settler and his sister. ${ }^{2}$

To date, most published accounts have focused largely upon the mucocutaneous lesions, though epilepsy, ${ }^{3}$ rage attacks and memory impairment ${ }^{4}$ have been noted. We now report two patients in whom paranoid symptoms were the presenting feature. In addition, each of them had a longstanding impairment of memory and typical intracranial calcifications.

\section{Case reports}

Case 1

A 51-year-old white male was referred to the Psychiatry Out-patient Department, Tygerberg Hospital with a history of altered behaviour over the past few years. His wife reported that he had become increasingly mistrustful and

Address for reprint requests: Dr RA Emsley, Department of Psychiatry, Faculty of Medicine, University of Stellenbosch, PO Box 63, Tygerberg 7505, Cape, South Africa.

Received 11 December 1984. Accepted 14 January 1985 suspicious and that he would make unreasonable demands. For example, he insisted that his family wear raincoats inside the house when it rained. The patient had been aware of impaired memory for at least 10 years, as a result of which he had been declared unfit for his work as a senior police officer. He was unable to accept this medical opinion and believed that a conspiracy was preventing him from obtaining alternative employment. His past history was noteworthy for hoarseness since childhood and brief episodes of interrupted consciousness years ago. These had never recurred, and he had not been prescribed anticonvulsants for the past 10 years. Of his three siblings, one brother apparently has a poor memory and has experienced episodic "absences". No further details of these problems could be obtained.

When examined he was alert and correctly orientated. Although no definite delusions were elicited, an extremely suspicious and aggressive attitude was noted. There were no hallucinations. Formal psychometric testing recorded average intellectual abilities (South African Wechsler Adult Intelligence Scale score 101), with selective, moderate to severe short-term memory impairment (Wechsler Memory Scale, Form 1, Memory selection A and B total score $3 \frac{1}{2}$ out of maximum score of 23 ). Physical examination revealed scarring of the angles of the mouth, neck and fingers. There were yellow papular lesions on the lower lip, soft palate and pharynx. The remainder of the systemic examination was unremarkable. Routine laboratory values, including random glucose, and tests of thyroid function were normal. A serological test for syphilis (VDRL) and tests for urinary and stool porphyrins were negative. An electroencephalogram was normal. An unenhanced CT scan showed bilateral symmetrical calcification in the region of the medial temporal lobes. These appearances would be consistent with involvement of the amygdaloid nucleus and possibly the communicating grey matter between this nucleus and the hippocampal uncus. 
Case 2

An 18-year-old female of mixed descent was admitted to the Psychiatric ward of Tygerberg Hospital with a 2 day history of abnormal behaviour. Since childhood, her voice had been hoarse and raised lesions had been present on her face and around her mouth. In addition, she later admitted to a poor memory of long standing. Her past health had been otherwise good, in particular there was no history of psychiatric illness or seizures. She had failed to pass one of nine years schooling and had been unable to maintain employment. Of four siblings, one was affected with hoarseness and skin lesions of a similar nature.

On admission, she was alert and agitated. Her speech was incoherent at times, but she appeared to be orientated. Persecutory delusions and verbal hallucinations were present. Physical examination revealed extensive acneform facial lesions and scarring, with fine nodules along the palpebral fissures. Her eyelashes were sparse. Yellow-white papules were seen on the inside of the lips and tongue. The tongue appeared enlarged, and had a "woody" consistency to palpation. Laryngeal infiltration was observed. The remainder of the systemic examination was unremarkable. Routine laboratory values, including random glucose, were normal. A serological test for syphillis (TPHA) and urine and blood screening tests for porphyria were negative. An electroencephalogram was normal. Skull radiographs showed intracranial calcification in the suprasellar region. An unenhanced CT scan (fig) demonstrated that this calcification was located in both temporal lobes, anteromedial to the tip of the inferior horn of the lateral ventricle. She was treated with chlorpromazine $150 \mathrm{mg}$ daily and supportive psychotherapy. Within 5 days her mental state returned to normal. A diagnosis of brief reactive psychosis was made, with social isolation, unemployment and an argument with her sister being considered responsible factors. Psychometric assessment after discharge recorded borderline mental retardation (Raven's Standard Progressive Matrices raw score 8 , Fick's Individual Scale of General Intelligence for South Africa raw score 55). Immediate associative memory was grossly impaired (Benton Visual Retention Test administration $\mathrm{A}$, form $\mathrm{C}$ : obtained correct 0 , and obtained errors 24 ) in the face of relatively intact visuo-motor abilities (Bender Visual Motor Gestalt Test raw score 6).

\section{Discussion}

Both of our patients manifested typical features of lipoid proteinosis, namely hoarseness since childhood, characteristic mucocutaneous lesions and pathognomonic intracranial calcifications. In addition, each of them had prominent impairment of memory out of proportion to intelligence. Such a restricted amnesic syndrome would be consistent with bilateral medial temporal lobe lesions. ${ }^{5}$ In
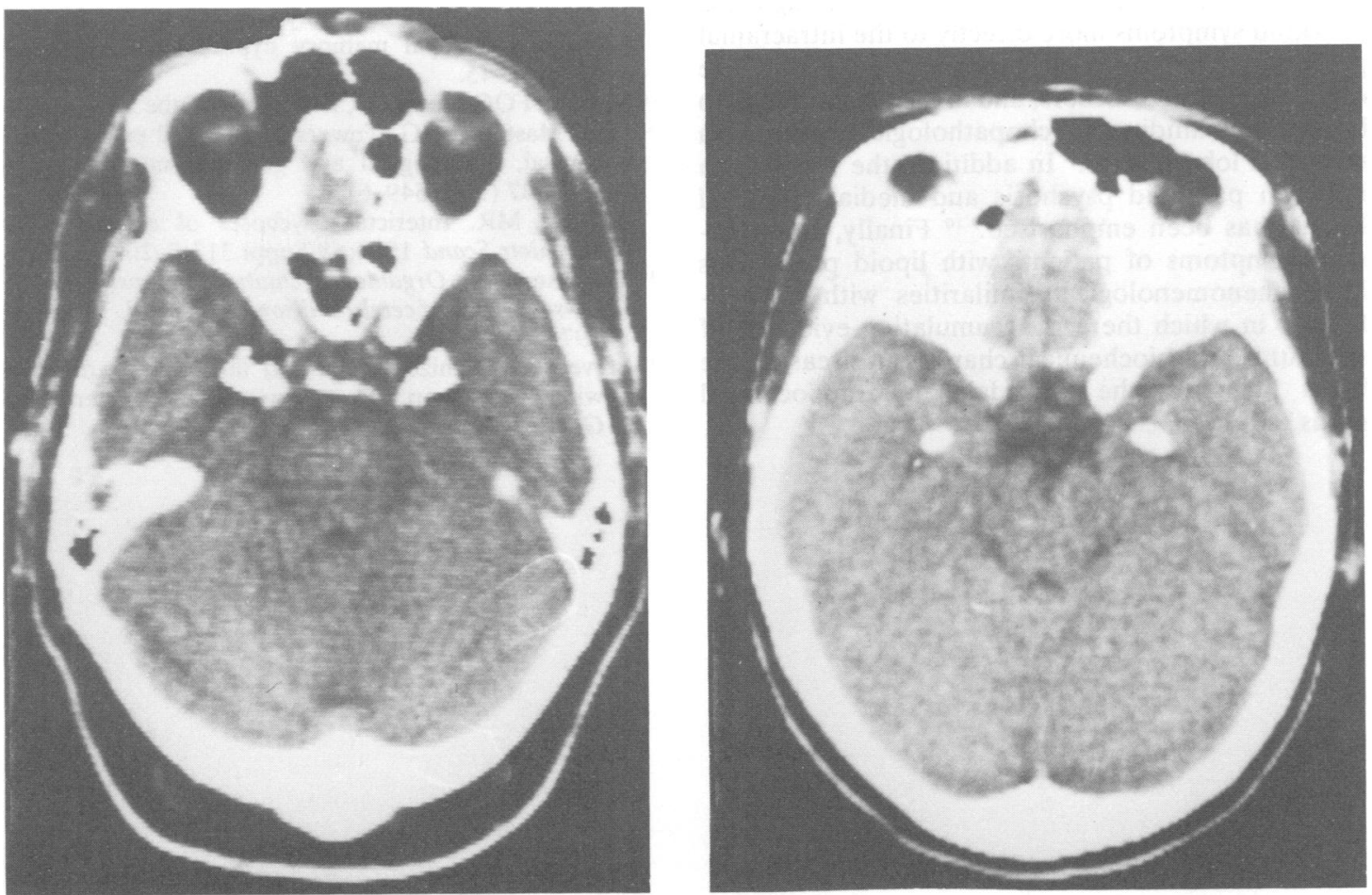

Fig CT scan of case 1 (left) and case 2 (right) showing the typical bilateral, symmetrical oval calcification in the anteromedial aspect of both temporal lobes. 
terms of the hippocampal memory hypothesis this would imply that the lesions involve both hippocampi. ${ }^{56}$ However, CT scan appearances show that the calcification did not involve these structures. In addition, in one of two other reported cases with significant memory impairment, pneumoencephalography was performed and showed that the hippocampi were not involved. ${ }^{4}$ These findings would seem to support an alternative proposal that medial amnesia results not from damage to the hippocampus, but rather to the temporal cortex or temporal stem. ${ }^{7}$ However, hippocampal involvement in these patients cannot be ruled out: necropsy findings of a patient with lipoid proteinosis and intracranial calcification showed that the lesions were more widely distributed than apparent macroscopically. The calcified areas consisted of dense amorphous masses of calcium and multiple haphazardly arranged small blood vessels, and marked proliferation of astrocytes and dense gliosis were present in adjacent tissue. ${ }^{8}$

In addition to our two patients, paranoid symptoms have been reported in one other. ${ }^{4}$ In psychodynamic terms, such symptoms could be interpreted as a psychological reaction to physical disfigurement and developing memory impairment. Alternatively, there are reasons for attributing these paranoid symptoms more directly to the intracranial pathology. First, paranoid thinking is often a feature of organic brain disorders, and in particular seems to be the outstanding psychopathological feature in temporal lobe lesions. ${ }^{9}$ In addition, the association between paranoid psychosis and medial temporal lesions has been emphasised..$^{910}$ Finally, the paranoid symptoms of patients with lipoid proteinosis have phenomenological similarities with schizophrenia, in which there is accumulating evidence of structural and biochemical changes in areas of the brain including the amygdala, parahippocampal gyrus and hippocampus. ${ }^{11}$
We are grateful to Dr RF Gledhill of the Institute of Psychiatry for his helpful advice and criticism of the manuscript. We also thank Dr RH Hewlett of the department of neuropathology for assisting in interpreting the CT scans, and Miss $\mathrm{T}$ Middleton for advice regarding the psychometric tests.

\section{References}

${ }^{1}$ Hofer PA. Urbach-Wiethe disease: a review. Acta Derm Venereol 1973;53:Suppl 71.

${ }^{2}$ Heyl T. Genealogical study of lipoid proteinosis in South Africa. Br J Derm 1970;83:338-40.

${ }^{3}$ Heyl T. Lipoid proteinosis: The clinical picture. $\mathrm{Br} J$ Derm 1963;75:465-72.

${ }^{4}$ Newton FH, Rosenberg RN, Lampert PW, O'Brein JS. Neurologic involvement in Urbach-Wiethe's disease (lipoid proteinosis). Neurology (Minneap) 1971;21: 1205-13.

s Scoville WB, Milner B. Loss of recent memory after bilateral hippocampal lesions. J Neurol Neurosurg Psychiatry 1957;20:11-21.

${ }^{6}$ Milner B. Amnesia following operation on the temporal lobes. In: Whitty CWM, Zangwill O, eds. Amnesia. London: Butterworths, 1966; ch 5: 109-33.

${ }^{7}$ Horel JA. The neuroanatomy of amnesia. A critique of the hippocampal memory hypothesis. Brain 1978; 101:403-45.

${ }^{8}$ Meenan FOC, Bowe SD, Dinn JJ, McCabe M, McCullen O, Masterson JG, Towers RP. Lipoid proteinosis; a clinical, pathological and genetic study. $Q \mathrm{~J}$ Med 1978;47 (188): 549-61.

${ }^{9}$ Trimble MR. Interictal psychoses of epilepsy. Acta Psychiatr Scand 1984;69:Suppl 313;9-20.

${ }^{10}$ Lishman WA. Organic psychiatry. The psychological consequences of cerebral disorder. Oxford: Blackwell, 1978:105-8.

"Stevens JR. Schizophrenia and the brain at the 1984 winter workshop, Davos, Switzerland (Letter). Arch Gen Psychiatry 1984;41:816-7. 